Tropical Journal of Pharmaceutical Research September 2020; 19 (9): 1827-1834

ISSN: $1596-5996$ (print); 1596-9827 (electronic)

(C) Pharmacotherapy Group, Faculty of Pharmacy, University of Benin, Benin City, 300001 Nigeria.

\title{
Rhodioloside inhibits apoptosis of hippocampal neurons exposed to sevoflurane via cAMP/PKA signaling pathway
}

\author{
Yuqiang Su, Yan Bai, Zhonglei Zheng, Xiaoying Fan* \\ The Second Affiliated Hospital of Xi'an Medical College, Department of Surgical Anesthesia, Xi'an,710038, China \\ *For correspondence: Email: dced7848@126.com; Tel: +8619654321022
}

Sent for review: 29 February 2020

Revised accepted: 4 May 2020

\begin{abstract}
Purpose: Neural injury affects patients after using inhalational anesthetics such as sevoflurane. Rhodioloside, a compound which is obtained from the Rhodiola rosea plant has been implicated to be the most commonly used psychostimulant that can improve a range of conditions. The study was aimed at finding the molecular mechanism underlying the Rhodioloside treatment of sevoflurane-injured hippocampal neurons.

Methods: Main hippocampal neurons, secluded from Sprague Dawley embryonic rats were employed to create an injury model using $3 \%$ sevoflurane. The sevoflurane-injured hippocampal neurons were treated with varying concentrations $(10,20,40$ and $80 \mu \mathrm{M} / \mathrm{ml})$ of Rhodioloside to create different experimental groups: RHSD10+SEV, RHSD20+SEV, RHSD40+SEV, RHSD80+SEV, while untreated cells were considered as the Control group. Cell viability was identified using the CCK-8 assay. The CFSE assay was used to verify the promotion function of Rhodioloside on cell differentiation of neurons. FCM assay was employed to determine cell proliferation and apoptosis. Expression levels of apoptosisrelated factors, like Caspase-3, Bcl-2 and Bax were examined by RT-qPCR, while Western blot was used to measure phosphorylation of PKA.

Results: Rhodioloside stimulated cell viability and prevented cell apoptosis in sevoflurane-injured hippocampal neurons in doses between 10-80 $\mu \mathrm{M}$. The apoptosis-inhibitory effect of Rhodioloside was observed to be through cAMP/PKA pathway activation. Also, expression levels of Bcl-2, and PKA were enhanced and the level of Caspase-3 and Bax was reduced in a dose-dependent pattern. The PKA inhibitor reversed the above observation in the $40 \mu \mathrm{M}$ Rhodioloside-treatment.

Conclusion: Rhodioloside promoted cell viability and prevented apoptosis of primary hippocampal neurons injured by sevoflurane, through cAMP/PKA pathway activation. Inhibition of PKA network deteriorated the function of Rhodioloside by stimulating cell apoptosis. Our findings present a novel evidence that Rhodioloside could attenuate neurotoxicity of inhalational anesthetics.
\end{abstract}

Keywords: Cell apoptosis, cAMP/PKA pathway, Hippocampal neurons, Rhodioloside, Sevoflurane

This is an Open Access article that uses a fund-ing model which does not charge readers or their institutions for access and distributed under the terms of the Creative Commons Attribution License (http://creativecommons.org/licenses/by/4.0) and the Budapest Open Access Initiative (http://www.budapestopenaccessinitiative.org/read), which permit unrestricted use, distribution, and reproduction in any medium, provided the original work is properly credited.

Tropical Journal of Pharmaceutical Research is indexed by Science Citation Index (SciSearch), Scopus, International Pharmaceutical Abstract, Chemical Abstracts, Embase, Index Copernicus, EBSCO, African Index Medicus, JournalSeek, Journal Citation Reports/Science Edition, Directory of Open Access Journals (DOAJ), African Journal Online, Bioline International, Open-J-Gate and Pharmacy Abstracts

\section{INTRODUCTION}

Sevoflurane is among the most used anesthetics in cesarean delivery and pediatric clinical process due to its swift induction and recovery features. Studies have shown over the years that the use of anesthesia in youngsters below 3 years could impact their development and behavior, thereby leading to cognitive disorders [1]. The critical period in the toxicity of anesthesia 
is dependent on the fast synaptogenesis, which continues from mid gestation to several years after birth [2]. Exposure of sevoflurane on immature brain, which could lead to neurotoxicity is associated with alterations in behavior of children later in adulthood and memory, reading, writing and mathematical learning challenges [1,3-6]. More studies further indicated that sevoflurane has the ability to induce neural apoptosis of a developing brain of a child $[7,8]$. It is essential to find a solution to anesthetic neurotoxicity, given that sevoflurane is used frequently in childbirth and surgeries to alleviate pain.

Rhodioloside, often referred to as salidroside is a compound found in the Rhodiola rosea (also known as golden root) plant and is one of the known potential compounds responsible for antidepressant and anxiolytic actions in plants [9]. The plant is a perennial herb in the family Crassulaceae and grows naturally in Artic Europe, including Britain, Asia and North America. It is abundantly and widely distributed in China. In Europe and Asia, R. rosea is mentioned as the most commonly used psychostimulant that can improve a range of conditions, including depression, anxiety, fatigue, anaemia, endurance, altitude sickness, insomnia and impotence $[10,11]$. A recent study found that the plant's supplement strikingly improves aerobic exercise performance after short-term high altitude training and has lots of biological activities, such as anti-stress and anti-fatigue $[12,13]$. Rhodiola exerts protective effects on chronic intermittent hypoxia-induced mitochondrial-dependent apoptosis in cardiac cells [14]. Accumulating evidence indicates that the plant extracts protect against cerebral ischemia-reperfusion injury in rat brain [15]. Pharmacological studies suggest that Rhodiola plant promotes cognitive function and relieves brain fatigue, clears reactive oxygen species, reduces oxidative stress, enhances physical endurance, ameliorates metabolic dysfunction, boost immunity and exerts anti-tumor effects [16]. In recent years, Rhodiola has attracted increasing attention because of its cognitive protective effects, antioxidant effects, and ability to scavenge reactive oxygen species. The potent component rhodioloside has been found to increase activation of the hippocampus and promote cell proliferation and differentiation of the neurons. Besides, rhodioloside improves neurogenesis in the hippocampus, hence boost cognition through regulation of apoptosis of the hippocampal neurons, hence prevents mental and memory death in scopolamin-treated rats [17]. There is however no clarity on how rhodioloside protects neurons from injury caused by sevoflurane.

Apoptosis is referred to as a programmed cell death and works by eliminating dysfunctional cells to keep up ordinary functions of body tissue. Anomalous apoptosis can lead to cell damage or loss of life [7]. Many reports show that sevoflurane triggers the stimulation of cysteine aspartate-specific protease (Caspace) and the apoptotic pathway [18]. Adenosine 3',5' cyclic monophosphate (CAMP) and CAMP/PKA, are the cAMP-dependent protein kinase (PKA) [19]. Recent studies have confirmed that differential effects of cAMP may be contributed to the features of PKA signaling pathway and impact positively on the neuroprotective process [20]. The antiapoptotic effect of ODN on neural cells is mediated through activation of the ODN metabotropic receptor, positively coupled to PKA, PKC and MAPK/ERK transduction pathways, which ultimately reduces the pro-apoptotic gene, Bax and stimulates $\mathrm{Bcl}-2$ expressions, and inhibits intracellular reactive oxygen species accumulation [21]. The sevoflurane neurotoxicity in not fully formed brain is associated with cAMP/PKA signaling pathway and other related factors leading to cell death, such as Caspace-3, B-cell lymphoma/leukemia-2 (Bcl-2) and Bcl-2 associated $\mathrm{X}(\mathrm{BaX})$. It is essential to understand the role of rhodioloside on sevoflurane-injured neurons via the cAMP/PKA pathway.

In this study, we therefore used the sevofluraneinjured rat hippocampal neuron and constructed its model to authenticate the likely role of rhodioloside on cell apoptosis triggered by sevoflurane. The role of CAMP/PKA signaling pathway was examined, which could be a potential clinical use of rhodioloside as a solution to anesthetic neurotoxicity.

\section{EXPERIMENTAL}

\section{Primary nerve cell cultures}

Matured female Sprague-Dawley rats, weighing 200 to $300 \mathrm{~g}$, were bought from Beijing Vital River Company, Beijing, China and kept for two weeks under typical environment of $22-24{ }^{\circ} \mathrm{C}$, 55-65 \% moisture in a 12-hour light/dark cycle. The process was carried out after breeding the female and male rats. The fetuses were then secluded for the study and an approval was obtained from the Institutional Animal Care and Use Committee of The Second Affiliated Hospital of Xi'an Medical College. The rats were sacrificed by cervical dislocation, wrapped up and sterilized using alcohol. After the sterilization of the uterus, the fetus was carefully removed. 
The hippocampus soft tissues were secluded and broken down by $0.125 \%$ trypsin for 30 min and nerve cells were gathered and cultured in Neural basal media, supplemented with $2 \%$ B27, $10 \mathrm{mmol} / 1 \mathrm{HEPES}$, and $0.5 \mathrm{mmol} / 1 \mathrm{~L}$ - glutamine in $5 \% \mathrm{CO}_{2}$-containing incubator at $37^{\circ} \mathrm{C}$. Cell culture media were altered every three days. Cell morphology was studied using DMi8 optical microscope after being cultured for 3 and 7 days, respectively.

After 7 days, the primary hippocampal neurons were pre-treated with Rhodioloside (RHSD) with varying concentrations $(10,20,40$ and $80 \mu \mathrm{M} / \mathrm{ml})$ for six hours, and injured by $3 \%$ sevoflurane gas assortment of $95 \%$ oxygen and $5 \%$ carbon dioxide; subsequently, the flow ratio was delivered at $2 \mathrm{l} / \mathrm{min}$. An injury model was constructed using $3 \%$ sevoflurane, a higher concentration than what is usually used in clinical application and scientific research. The experimental groups were termed: RHSD10+SEV, RHSD20+SEV, RHSD40+SEV, RHSD80+SEV, respectively. SEV group was given to cells that were treated with $3 \%$ sevoflurane gas alone, RHSD40 for cells treated with $40 \mu \mathrm{M} / \mathrm{ml} \mathrm{RHSD}$, and untreated cells were considered as the Control group. Besides, to have the finest repair outcome, RHSD40+SEV cells were selected to be treated with $5 \mu \mathrm{mol} / \mathrm{l}$ ERK1/2 inhibitor PD98059 $1 \mathrm{hr}$ before $40 \mu \mathrm{g} / \mathrm{ml}$ RHSD treatment (cAMP/PKA inhibitor+RHSD40 $+\mathrm{SEV}$ group). The changes were compared to that of the Control, SEV, RHSD40+SEV groups.

\section{Cell counting kit-8 assay}

Cell practicability of primary hippocampal neurons in the experimental groups (Control, RHSD40, SEV, RHSD10+SEV, RHSD20+SEV, RHSD40+SEV, LBP80+SEV) were assessed using the CCK-8 assay in dedicated times (2, 4 and $6 \mathrm{hr}$ ), based on the manufacturer's protocols. After the treatment, the cells were planted in 96-well plates and reacted with $20 \mu \mathrm{l} \mathrm{CCK}-8$ reagent for $1 \mathrm{hr}$ in $5 \% \mathrm{CO}_{2}$-containing incubator at $37^{\circ} \mathrm{C}$. The ophthalmic density standards were measured using the microplate reader at $450 \mathrm{~nm}$.

\section{Carboxyfluorescein diacetate succinimidyl ester (CFSE) assay}

Using the CFSE cell proliferation kit (Invitrogen, USA), the proliferation potential of cells in different clusters (Control, SEV, RHSD10+SEV, RHSD20+SEV, RHSD40+SEV groups) was examined. The assay was performed based on the manufacturer's instructions. Cells were resuspended to $1 \times 10^{6} / \mathrm{ml}$ in $1 \mathrm{ml}$ preheated phosphate buffer solution (PBS) in sterile centrifuge tubes. An aliquot of $2 \mu \mathrm{l}$ CFSE (5 $\mathrm{mmol} / \mathrm{l}$ ) stocking reagent was added into cell suspension to a final concentration of $10 \mu \mathrm{mol} / \mathrm{l}$. Cells were kept warm at $37^{\circ} \mathrm{C}$ for $10 \mathrm{~min}$ and then in $10 \mathrm{ml}$ icy culture media for $5 \mathrm{~min}$ in the dark. Finally, cells were inoculated in 24-well plates (1x105/well) and kept warm with $5 \% \mathrm{CO}_{2}$ at $37^{\circ} \mathrm{C}$, then cells were digested, collected and detected by FACS Calibur flow cytometer without light in the entire process.

\section{Apoptosis detection}

Apoptotic rates of hippocampal neurons in the experimental groups (Control, SEV, RHSD10+SEV, RHSD20+SEV, RHSD40+SEV groups) were determined by Annexin V/PI (propidine iodide) double-stain assay, according to the company's instructions (BioVision, Inc., Milpitas, CA, USA). Annexin V/FITC (fluorescein isothiocyanate) was used to measure the Phosphatidylserine exposed to the outside of cell membrane in early stage of apoptosis. PI also helped to detect DNA within membrane-injured cells in the late-stage of apoptosis. Cells were resuspended by $100 \mu \mathrm{l}$ binding buffer to a final concentration of $1 \times 10^{6}$ cells $/ \mathrm{ml}$. Then, $5 \mu \mathrm{l}$ Annexin V/FITC and $10 \mu \mathrm{PI}(20 \mu \mathrm{g} / \mathrm{ml})$ were added. After incubation for $15 \mathrm{~min}$ in darkness at room temperature, a flow cytometer (BD Biosciences) was used to analyze cells, with 488 $\mathrm{nm}$ as thrilling light wavelength, and $515 \mathrm{~nm}$ (FITC), $560 \mathrm{~nm}$ (PI) as spotting light wavelengths.

\section{Reverse Transcription-Quantitative Polyme- rase Chain Reaction (RT-qPCR)}

RT-qPCR was done to analyze mRNA expression levels of apoptosis-related factors such as Caspase-3, Bax, Bcl-2 in different groups, including Control, SEV, RHSD10+SEV, RHSD20+SEV, RHSD40+SEV and cAMP/PKA inhibitor+ RHSD40+SEV groups. GAPDH was used as an inner reference. Initially, TRIzol (Invitrogen; Thermo Fisher Scientific, Inc., Waltham, MA, USA) was used to extract total RNA and reversely transcribed to CDNA with a Paramount Strand cDNA kit (Takara Bio, Inc., Otsu, Japan), based on the manufacturer's instructions. PCR strengthening was carried out using the SYBR Premix Ex Taq kit (Takara Bio, Inc.). Briefly, after pre-denaturation at $95^{\circ} \mathrm{C}$ for 30 sec, amplification of 40 cycles were conducted: denaturation at $95^{\circ} \mathrm{C}$ for $5 \mathrm{sec}$, annealing/extension at $60^{\circ} \mathrm{C}$ for $30 \mathrm{sec}$, which was performed in $\mathrm{ABI} 7300$ Thermocycler (Applied Biosystems; Thermo Fisher Scientific, Inc.). The primer sequences were presented in Table 1.

Trop J Pharm Res, September 2020; 19(9): 1829 
Table 1: Nucleotide sequences of primers used for targeting genes

\begin{tabular}{|c|c|c|}
\hline Primer & Direction & Nucleotide sequence \\
\hline \multirow[t]{2}{*}{ Caspase-3 } & Sense & $\begin{array}{l}\text { 5'-TGG } \\
\text { AATGTCAGCTCGCAATG } \\
-3^{\prime}\end{array}$ \\
\hline & Antisense & $\begin{array}{l}\text { 5'-CAGGTC } \\
\text { CGTTCGTTCCAAAA-3' } \\
\text { 5'- }\end{array}$ \\
\hline \multirow[t]{2}{*}{ Bax } & Sense & $\begin{array}{l}\text { AGATCATGAAGACAGG } \\
\text { GGCC-3' }\end{array}$ \\
\hline & Antisense & $\begin{array}{l}\text { 5'-ATCCTCTGCAGCTCC } \\
\text { ATGTT-3' } \\
\text { 5'- }\end{array}$ \\
\hline \multirow[t]{2}{*}{$\mathrm{Bcl}-2$} & Sense & $\begin{array}{l}\text { AACTCTTCAGGGATGG } \\
\text { GG TG-3' }\end{array}$ \\
\hline & Antisense & $\begin{array}{l}\text { 5'- } \\
\text { GCTGGGGCCATATAGT } \\
\text { TCCA-3' }\end{array}$ \\
\hline \multirow{2}{*}{ GAPDH } & Sense & $\begin{array}{l}\text { 5'- } \\
\text { AGTCTACTGGCGTCTTC } \\
\text { ACC-3' }\end{array}$ \\
\hline & Antisense & $\begin{array}{l}\text { CCACGATGCCAAAGTT } \\
\text { GTCA-3' }\end{array}$ \\
\hline
\end{tabular}

\section{Western blot analysis}

Western blotting was conducted to determine protein levels, including apoptosis-interrelated factors (active-Caspase-3, Bax, Bcl-2) and p-PKA (phosphorylated PKA), t-PKA (total-PKA), in diverse clusters which include: Control, SEV, RHSD10+SEV, RHSD20+SEV, RHSD40+SEV and PKA inhibitor+RHSD40+SEV groups. Proteins were first acquired and the concentration was determined by BCA assay (Beyotime Institute of Biotechnology, China). Sodium dodecyl sulfate-polyacrylamide gel electrophoresis (SDS-PAGE) was then used to concentrate and separate the proteins, and electrotransferred onto a polyvinylidene fluoride (PVDF) membrane (EMD Millipore, Billerica, MA, USA). After the blockage with $5 \%$ nonfat dry milk for an hour, blotted membranes were incubated with specific crucial antibodies overnight at $4{ }^{\circ} \mathrm{C}$, respectively. GAPDH was used as loading control. The primary antibodies were as follows: anti-PKA (Abcam; ab17942, 1:1,000), anti-p-PKA (Cell Signaling Technology, Inc., Danvers, MA, USA; 4370, 1:2,000), anti-GAPDH (Abcam; ab9485, 1:2,500). After that, membranes were incubated with the appropriate secondary antibody goat anti-rabbit HRP-conjugated IgG H\&L (Abcam; ab6721, 1:5,000) for $2 \mathrm{hr}$. The PVDF membrane were exposed to and detected by X-ray film with enhanced chemiluminescense (ECL) detection system reagents (Amersham; GE Healthcare, Chalfont, UK).

\section{Statistical analysis}

All the functional assays were repeated three times. All data were presented as mean \pm standard deviation of three independent replicates. Statistical analysis was conducted by means of a SPSS 22.0 statistical package (IBM Corp., Armonk, NY, USA), and Welch's one-way analysis of variance (ANOVA) was used to analyze the dissimilarities among multi-groups. Both Games-Howell test was also employed. $\mathrm{P}<0.05\left({ }^{* *}\right)$ and $\mathrm{P}<0.01\left(^{* * *}\right)$ were considered significant.

\section{RESULTS}

\section{Rhodioloside promotes cell viability of hippocampal neurons injured by sevoflurane}

Hippocampal primary neurons were isolated from SD embryonic rats. CCK-8 assay was used to detect the function of rhodioloside with different concentrations $(10,20,40$ and $80 \mu \mathrm{M} / \mathrm{ml})$ on cell viability of hippocampal primary neurons treated with $3 \%$ sevoflurane, at $6 \mathrm{hr}$. The function of 40 $\mu \mathrm{M} / \mathrm{ml}$ RHSD alone was also detected. RHSD alone had no effect on neurons viability. Cell viability of neurons decreased significantly with sevoflurane treatment, compared with control group $(P<0.01)$. Meanwhile, cell viability of sevoflurane-injured neurons increased significantly by RHSD in dose- $(10,20,40$ and 80 $\mu \mathrm{M} / \mathrm{ml}$ ) dependent manner, compared with SEV group $(P<0.05)$ (Figure 1A). The effect of 80 $\mu \mathrm{M} / \mathrm{ml}$ was similar to that of $40 \mu \mathrm{M} / \mathrm{ml} \mathrm{RHSD}$ (Fig.1A). Therefore, we excluded the $80 \mu \mathrm{M} / \mathrm{ml}$ RHSD group for the following assays. CFSE assay was conducted to verify the promotion function of RHSD with different concentrations $(10,20$ and $40 \mu \mathrm{M} / \mathrm{ml})$ on cell proliferative ability of hippocampal primary neurons treated by $3 \%$ sevoflurane for $6 \mathrm{hr}$, showing that cell proliferative ability of neurons was inhibited significantly, with decreased M1 values, by sevoflurane in SEV group, compared with control group $(P<0.01)$, which was promoted significantly, with increased M1 values, by RHSD in dose-dependent manners $(10,20$ and 40 $\mu \mathrm{M} / \mathrm{ml})(\mathrm{P}<0.05)$ (Figure 1B).

\section{Rhodioloside inhibited cell apoptosis of sevoflurane-injured hippocampal neurons}

Annexin V/PI double-stain and FCM assay were done to determine the effect of RHSD with different concentrations $(10,20$ and $40 \mu \mathrm{M} / \mathrm{ml})$ on cell apoptotic status of hippocampal neurons injured by $3 \%$ sevoflurane for $6 \mathrm{hr}$. The results presented in Figure $2 \mathrm{~A}$ suggested that sevoflurane remarkably facilitated cell apoptosis 
of neurons in SEV group, compared with control group $(P<0.01)$, which was reduced significantly by RHSD in dose-dependent manners $(10,20$ and $40 \mu \mathrm{M} / \mathrm{ml})(P<0.05)$. Expression levels of apoptosis-related factors, such as Caspase-3, $\mathrm{Bax}, \mathrm{Bcl}-2$, were determined by RT-qPCR in the experimental groups. The results demonstrated that sevoflurane dramatically up-regulated the expression levels of pro-apoptotic factors, Caspase-3 and Bax $(P<0.01)$, and RHSD effectively down-regulated them dose-dependently $(10,20$ and $40 \mu \mathrm{M} / \mathrm{ml})$, both in mRNA and protein manners $(P<0.05)$. Meanwhile, sevoflurane notably down-regulated the expressions of apoptotic inhibitor, Bcl-2 $(P<0.01)$, and RHSD effectively up-regulated them, dose-dependently $(10,20$ and $40 \mu \mathrm{M} / \mathrm{ml})$, both in mRNA and protein manners $(P<0.05$; Figure 2B).

\section{Rhodioloside activated cAMP/PKA signaling pathway in sevoflurane-injured hippocampal neurons}

The phosphorylation levels of cAMP/PKA in the experimental groups were assessed by Western blot. The results indicated that, protein levels of p-PKA decreased significantly in SEV group, compared with the control group $(P<0.01)$, which increased notably dose-dependently $(10,20$ and $40 \mu \mathrm{M} / \mathrm{ml}$ ) when treated with RHSD, compared with SEV group $(P<0.05)$. Meanwhile, there was no significant difference in t-PKA levels among different experimental groups (Figure $3 \mathrm{~A}$ and $\mathrm{B}$ ).

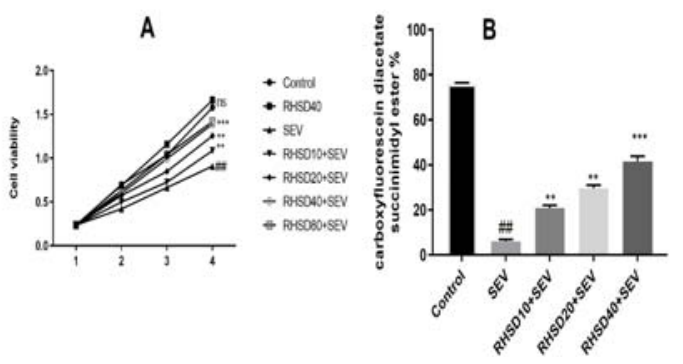

Figure 1: Rhodioloside promoted cell viability of hippocampal neurons injured by sevoflurane. (A) CCK 8 assay was used to detect function of RHSD with different concentrations (10, 20, 40 and $80 \mu \mathrm{M} / \mathrm{ml})$ on cell viability of $3 \%$ sevoflurane treated neurons in different groups (Control, RHSD40, SEV, RHSD10+SEV, RHSD20+SEV, RHSD40+SEV, RHSD80+SEV). (B) CFSE assay was conducted to verify the promotion function of RHSD on cell differentiation of neurons, after $6 \mathrm{hr}$ treatment of sevoflurane. \#\#: $P<0.01$ vs. Control group; **: $P<0.05$; ***: $P<0.01$ vs. SEV group. RHSD: Rhodiolosides; SD: Sprague-Dawley; CCK-8: Cell Counting Kit-8; CFSE: carboxyfluorescein diacetate succinimidyl ester

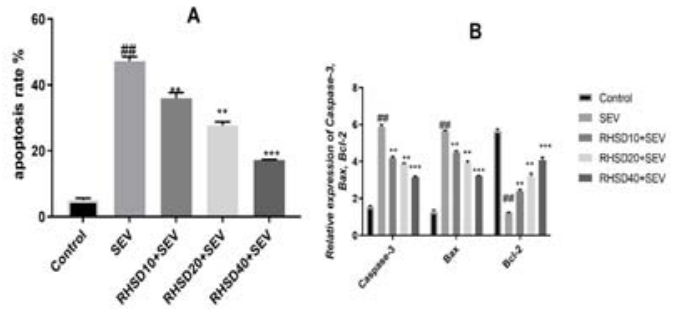

Figure 2: Rhodioloside inhibited cell apoptosis of sevoflurane- injured hippocampal neurons. (A) Annexin V/PI double-stain and FCM assay were performed to evaluate the effect of RHSD with different concentrations (10, 20 and $40 \mu \mathrm{M} / \mathrm{ml})$ on cell apoptosis of neurons injured by $3 \%$ sevoflurane for 6 hr. (B) Expression levels of apoptosis-related factors, such as Caspase-3, Bax, Bcl-2, were determined by RT-qPCR. \#: $P<0.01$ vs. Control group; *: $P<0.05$; **: $P<0.01$ vs. SEV group; RHSD: Rhodioloside; FCM: flow cytometry

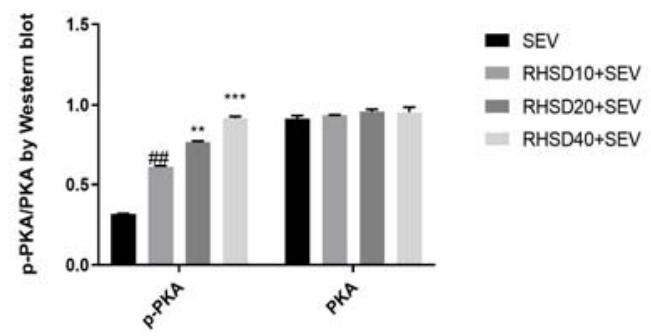

Figure 3: Rhodioloside activated cAMP/PKA signaling pathway in hippocampal neurons injured by sevoflurane. The relative phosphorylation levels of PKA were assessed by Western blot in Control, SEV, RHSD10+SEV, RHSD20+SEV, RHSD40+SEV groups. \#\#: $P<0.01$ vs. Control group; **: $P<0.05$; ***: $P<0.01$ vs. SEV group; RHSD: Rhodioloside

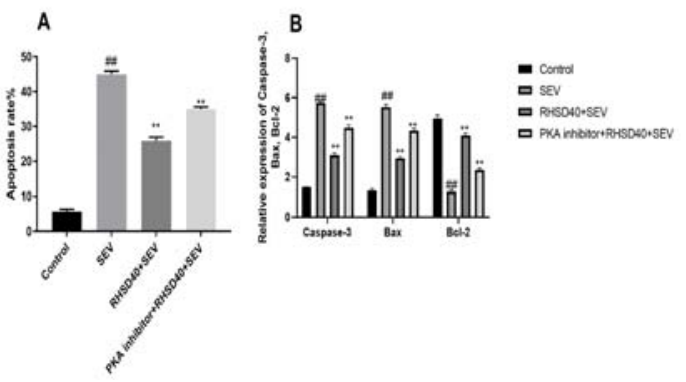

Figure 4: Rhodioloside inhibited cell apoptosis through cAMP/PKA signal pathway in sevofluraneinjured hippocampal neurons. (A) FCM analysis was conducted to analyze the apoptotic rates in the experimental groups. (B) RT-qPCR analysis was performed to analyze expressions of pro-apoptotic cytokines in Control, SEV, RHSD40+SEV, PKA inhibitor+ RHSD40+SEV groups. \#\#: $P<0.01$ vs. Control group; **: $P<0.01$ vs. SEV group, vs. RHSD40+SEV group; RHSD: Rhodioloside 
The function of rhodioloside was blocked by cAMP/PKA inhibitor in sevoflurane-injured hippocampal neurons

The FCM analysis on apoptotic rates verified that, RHSD decreased the apoptotic rates of hippocampal neurons treated with sevoflurane in RHSD40+SEV group, which was reversed by PKA inhibitor $(P<0.05)$ (Figure 4A). RT-qPCR assays showed that the levels of active Caspase- 3 and Bax were promoted and $\mathrm{Bcl}-2$ was inhibited by PKA inhibitor in PKA inhibitor+RHSD40+SEV group, compared with RHSD40+SEV group $(P<0.05)$ (Figure 4B).

\section{DISCUSSION}

Sevoflurane has been found to regularly cause behavioral and developmental cognitive maladies in children, which has necessitated research into finding remedies to avert its developmental anesthetic neurotoxicity [22,23]. Rhodiolosides are effectual to heighten neurogenesis in hippocampus and enhance cognition via apoptosis regulation in hippocampal neurons under stress. Verifying the likely molecular means of rhodioloside shielding neurons from sevoflurane injury is an exciting practice. The hippocampus is the fundamental tissue for spatial navigation and long-term memory in the brain. Sevoflurane (3\%) was used to develop an injury model in previous studies. Cell differentiation and the entire process of neuron development occurred together. In the current study, we found that rhodioloside promoted cell viability and prevented cell apoptosis of neurons damaged by $3 \%$ sevoflurane dose-dependently (10, 20 and $40 \mu \mathrm{M} / \mathrm{ml}$ ).

Sevoflurane neurotoxicity in undeveloped brain is linked to extreme apoptosis, including anomalous expression of $\mathrm{Bcl}-2$ family members and caspases [18]. The members of Bcl-2 family, including $\mathrm{Bax}, \mathrm{Bcl}-2$ and $\mathrm{Bcl}-\mathrm{XL}$, are the primary apoptosis regulators [24, 25]. As an anti-apoptotic factor, Bcl-2 prevents apoptosis mostly by barring caspase pathway. On the contrary, Bax conjugates with Bcl-2, forms heterodimer to stimulate the discharge of cytochrome, activates caspase path and fosters apoptosis $[26,27]$. Caspases are a family of cysteine-aspartic proteases. Being the final executor of caspase pathway, caspase- 3 plays an important role to cut down cellular components and induces apoptosis [28]. The findings of this study supported the results of other experiments by proving that sevoflurane-treatment on hippocampal neurons significantly increased the levels of expression of pro-apoptotic factors, such as Bax and
Caspase-3, and reduced the levels of expression of anti-apoptotic factors such as Bcl-2. According to the findings, rhodioloside enhanced cell viability and differentiation, prevented apoptosis dose-dependently by weakening $\mathrm{Bcl}-2$ and increasing Bax and Caspase- 3 expression, in sevoflurane-injured hippocampal neurons, as expected.

Recent studies have proven that several pathways were involved in the injury of neurons by sevoflurane. The cAMP/PKA pathway is activated by a diverse stimulus, including growing factors, mechanical stress and so on. For signal transduction occurrence from the surface reflectors of cell membrane to the nucleus, PKA should be activated. According to research, cAMP/PKA signaling pathway plays a crucial role in the developmental neuronal endurance and sevoflurane neurotoxicity [6-8], to increase dendritic spine density during synaptogenesis in hippocampal neurons, and to help enhance learning and retention abilities. In this study, rhodioloside was discovered to promote cAMP/PKA activation effectively by increasing the levels of PKA phosphorylation, which was extraordinarily reduced by sevoflurane in hippocampal neurons.

PKA inhibitor lessened the function of rhodioloside on apoptosis inhibition in hippocampal neurons treated with sevoflurane. Consistent with the promoted apoptosis, the expression levels of Bax and active-Caspase increased, and $\mathrm{Bcl}-2$ decreased when PKA inhibitor was present. Though, it would be better to examine the effect of PKA activation on it at the same time, the results have indicated that rhodioloside protected neurons from sevoflurane induced apoptosis through cAMP/PKA pathway, which might reduce cell viability and proliferative ability of primary hippocampal neurons.

\section{CONCLUSION}

The findings from this study suggest that rhodioloside promotes cell viability and proliferation, and prevents apoptosis of primary hippocampal neurons injured by sevoflurane, through CAMP/PKA pathway activation. Moreover, addition of PKA inhibitor deteriorated the function of rhodioloside by stimulating cell apoptosis. Our findings provided a novel evidence that rhodioloside could attenuate neurotoxicity of inhalational anesthetics in the clinics. The future direction to this study will be to investigate the effect of rhodioloside on sevoflurane-induced behavioral deviations, cognitive and memorial disorders in vivo.

Trop J Pharm Res, September 2020; 19(9): 1832 


\section{DECLARATIONS}

\section{Conflict of interest}

No conflict of interest is associated with this work.

\section{Contribution of authors}

We declare that this work was done by the authors named in this article and all liabilities pertaining to claims relating to the content of this article will be borne by the authors.

\section{Open Access}

This is an Open Access article that uses a funding model which does not charge readers or their institutions for access and distributed under the terms of the Creative Commons Attribution License (http://creativecommons.org/licenses/by/ 4.0) and the Budapest Open Access Initiative (http://www.budapestopenaccessinitiative.org/rea d), which permit unrestricted use, distribution, and reproduction in any medium, provided the original work is properly credited.

\section{REFERENCES}

1. Dobbing J, Sands J. Comparative aspects of the brain growth spurt. Early Hum Dev, 1979; 3:79-83.

2. Yon JH, Daniel-Johnson J, Carter LB, Jevtovic-Todorovic $V$. Anesthesia induces neuronal cell death in the developing rat brain via the intrinsic and extrinsic apoptotic pathways. Neurosci. 2005; 135: 815-27.

3. DiMaggio C, Sun LS, Kakavouli A, Byrne MW, Li, G. A retrospective cohort study of the association of anesthesia and hernia repair surgery with behavioral and developmental disorders in young children," J Neurosurg Anesthesiol. 2009;21: 286-91.

4. Wilder RT, Flick RP, Sprung J, Katusic SK, Barbaresi WJ, Mickelson C, et al. Early exposure to anesthesia and learning disabilities in a population-based birth cohort. Anesthesiol. 2009;110; 796-804.

5. Servick K. Biomedical Research. Researchers struggle to gauge risks of childhood anesthesia. Sci. 2014; 346:1161-2.

6. Edwards DA, Shah HP, Cao W, Gravenstein N, Seubert $C N$, Martynyuk AE. Bumetanide alleviates epileptogenic and neurotoxic effects of sevoflurane in neonatal rat brain. Anesthesiol. 2010;112;567-75

7. Lu Y, Wu X, Dong $Y, X u X$, Zhang $Y$, Xie Z. Anesthetic sevoflurane causes neurotoxicity differently in neonatal naive and Alzheimer disease transgenic mice," Anesthesiol. 2010;112,1404-16.

8. Satomoto M, Satoh $Y$, Terui K, Miyao H, Takishima K, Ito $N$ et al. Neonatal exposure to sevoflurane induces abnormal social behaviors and deficits in fear conditioning in mice. Anesthesiol. 2009;110;628-37.

9. Zhang $X, D u$ Q, Liu C, Yang Y, Wang J, Duan S et al. Rhodioloside ameliorates depressive behavior via upregulation of monoaminergic system activity and antiinflammatory effect in olfactory bulbectomized rats. Int Immunopharmacol. 206;36;300-304.

10. Hung SK, Perry R, Ernst. The effectiveness and efficacy of Rhodiola rosea L.: a systematic review of randomized clinical trials. Phytomed. 2011; 18:235-44.

11. Panossian A, Hambardzumyan $M$, Hovhanissyan $A$, Wikman G. The adaptogens rhodiola and schizandra modify the response to immobilization stress in rabbits by suppressing the increase of phosphorylated stressactivated protein kinase, nitric oxide and cortisol. Drug Target Insights. 2007; 2:39-54.

12. L Ma, DL Cai, HX Li, BD Tong, Y Wang, and SP Pei. "[Protective effects of salidroside on oxidative damage in fatigue mice]," Zhong Xi Yi Jie He Xue Bao, vol. 7, pp. 237-41, Mar 2009.

13. SC Huang, FT Lee, TY Kuo, JH Yang, and CT Chien. "Attenuation of long-term Rhodiola rosea supplementation on exhaustive swimming-evoked oxidative stress in the rat," Chin J Physiol, vol. 52, pp. 316-24, Oct 312009.

14. $R$ Yan, $H X u$, and $X F u$. "Salidroside protects hypoxiainduced injury by up-regulation of miR-210 in rat neural stem cells," Biomed Pharmacother, vol. 103, pp. 14901497, Jul 2018.

15. ZQ Qu, Y Zhou, YS Zeng, YK Lin, Y Li, ZQ Zhong, et al. "Protective effects of a Rhodiola crenulata extract and salidroside on hippocampal neurogenesis against streptozotocin-induced neural injury in the rat," PLoS One, vol. 7, p. e29641, 2012.

16. SF Nabavi, N Braidy, IE Orhan, A Badiee, M Daglia, and SM Nabavi. "Rhodiola rosea $L$. and Alzheimer's Disease: From Farm to Pharmacy," Phytother Res, vol. 30, pp. 532-9, Apr 2016.

17. L Chen, P Liu, X Feng, and C Ma. "Salidroside suppressing LPS-induced myocardial injury by inhibiting ROS-mediated PI3K/Akt/mTOR pathway in vitro and in vivo," J Cell Mol Med, vol. 21, pp. 3178-3189, Dec 2017.

18. X Zhang, Z Xue, and A Sun. "Subclinical concentration of sevoflurane potentiates neuronal apoptosis in the developing C57BL/6 mouse brain," Neurosci Lett, vol. 447, pp. 109-14, Dec 122008.

19. BS Skalhegg and $K$ Tasken. "Specificity in the cAMP/PKA signaling pathway. Differential expression, regulation, and subcellular localization of subunits of PKA," Front Biosci, vol. 5, pp. D678-93, Aug 12000.

20. $R$ Guan, J Lv, F Xiao, Y Tu, Y Xie, and L Li. "Potential role of the $C A M P / P K A / C R E B$ signalling pathway in hypoxic preconditioning and effect on propofol-induced neurotoxicity in the hippocampus of neonatal rats," Mol Med Rep, vol. 20, pp. 1837-1845, Aug 2019.

21. O Masmoudi-Kouki, Y Hamdi, I Ghouili, S Bahdoudi, H Kaddour, J Leprince, et al. "Neuroprotection with the 
Endozepine Octadecaneuropeptide, ODN," Curr Pharm Des, vol. 24, pp. 3918-3925, 2018.

22. SY Li, D Yang, CM Yeung, WY Yu, RC Chang, KF So, et al. "Lycium barbarum polysaccharides reduce neuronal damage, blood-retinal barrier disruption and oxidative stress in retinal ischemia/reperfusion injury," PLoS One, vol. 6, p. e16380, Jan 262011.

23. H Li, Y Liang, K Chiu, Q Yuan, B Lin, RC Chang, et al. "Lycium barbarum (wolfberry) reduces secondary degeneration and oxidative stress, and inhibits JNK pathway in retina after partial optic nerve transection," PLoS One, vol. 8, p. e68881, 2013.

24. JW Park, MS Lim, SY Ji, MS Cho, SJ Park, SH Han, et al. "Effects of short-term exposure to sevoflurane on the survival, proliferation, apoptosis, and differentiation of neural precursor cells derived from human embryonic stem cells, "J Anesth, vol. 31, pp. 821-828, Dec 2017.

25. AB Ozer, S Ceribasi, AO Ceribasi, I Demirel, MK Bayar, $B$ Ustundag, et al. "Effects of sevoflurane on apoptosis,
BDNF and cognitive functions in neonatal rats," Bratisl Lek Listy, vol. 118, pp. 80-84, 2017.

26. $X$ Yang, S Yang, C Hong, $W Y$ Y, and $W$ Guonian> "Panax Notoginseng Saponins attenuates sevofluraneinduced nerve cell injury by modulating AKT signaling pathway," Mol Med Rep, vol. 16, pp. 7829-7834, Nov 2017.

27. ML Ding, H Ma, YG Man, and HY Lv. "Protective effects of a green tea polyphenol, epigallocatechin-3-gallate, against sevoflurane-induced neuronal apoptosis involve regulation of CREB/BDNF/TrkB and PI3K/Akt/mTOR signalling pathways in neonatal mice," Can J Physiol Pharmacol, vol. 95, pp. 1396-1405, Dec 2017.

28. H Huang, CM Liu, J Sun, WJ Jin, YQ Wu, and J Chen. "Repeated 2\% sevoflurane administration in 7 and 60 day-old rats: Neurotoxicity and neurocognitive dysfunction," Anaesthesist, vol. 66, pp. 850-857, Nov 2017. 